\title{
Características de pacientes com indicação de cuidados paliativos em uma unidade de cuidados prolongados em Campo Grande, Mato Grosso do Sul
}

\author{
Characteristics of patients with palliative care indication in \\ an extended care unit in Campo Grande, Mato Grosso do Sul
}

Características de los pacientes con indicación de cuidados paliativos en una unidad de cuidados extendidos en Campo Grande, Mato Grosso do Sul

${\text { Anna Alice Vidal Bravalhieri }{ }^{1}}^{\text {Suzi Rosa Miziara Barbosa }}{ }^{2}$
Maria de Fátima Bregolato Rubira de Assis ${ }^{3}$
Ramon Moraes Penha ${ }^{4}$

${ }^{1}$ Mestranda em Saúde e Desenvolvimento na Região Centro-Oeste, pela Universidade Federal de Mato Grosso do Sul. Especialista pelo Programa de Residência Multiprofissional em Cuidados Continuados Integrados - Atenção à Saúde do Idoso. Graduação em Fisioterapia pela Universidade Católica Dom Bosco. E-mail: aabravalhieri@gmail.com, Orcid: http://orcid.org/0000-0002-5512-7338

${ }^{2}$ Doutora em Ciências da Saúde pela Universidade Federal de Uberlândia. Mestre em Fisioterapia pelo Centro Universitário do Triângulo. Graduada em Fisioterapia pela Universidade Católica de Petrópolis. Docente no Curso de Fisioterapia da Universidade Federal de Mato Grosso do Sul. E-mail: srmiziara@gmail.com, Orcid: http://orcid.org/0000-0003-4946-9726

${ }^{3}$ Mestre em Meio Ambiente e Desenvolvimento Regional pela Universidade Anhanguera-Uniderp. Especialista em Metodologia e Gestão para Educação

a Distância; Desenvolvimento de Recursos Humanos para a Qualidade; Administração Hospitalar; Gestão de Serviços de Saúde. Graduada em Serviço Social pela Faculdade Integrada de Marília. Coordenadora do Programa de Residência Multiprofissional em Cuidados Continuados Integrados. E-mail: fatimabrubira@gmail.com, Orcid: http://orcid.org/0000-0002-2332-5815

${ }^{4}$ Doutor e mestre em Ciências pelo Programa de Pós-Graduação em Enfermagem na Saúde do Adulto, da Escola de Enfermagem da Universidade de São Paulo. Especialista em Gerontologia pela Universidade Federal de Santa Catarina. Graduado em Enfermagem pela Universidade Estadual de Mato Grosso do Sul (UEMS). Docente na UEMS. E-mail: rvamus@usp.br, Orcid: http://orcid.org/0000-0002-8656-2535 
Resumo: Cuidado paliativo (CP) é uma abordagem que promove alívio de sofrimento de pessoas com doenças incuráveis que ameaçam a continuidade da vida. O objetivo deste estudo foi traçar as características dos pacientes com indicação de CP em uma unidade de cuidados prolongados (UCP). Trata-se de um estudo transversal, quantitativo, retrospectivo e com coleta de dados em prontuário. No período do estudo, houve 389 admissões na UCP e, dessas, 31 prontuários foram incluídos para este estudo. Destacou-se a população idosa, compondo mais de $80 \%$ da amostra; $54,8 \%$ eram mulheres, acometidas principalmente por doenças crônicas relacionadas ao processo de envelhecimento, como as doenças neurodegenerativas e com internação de curto prazo; e 84\% foram a óbito. Foi observada a necessidade de fortalecimento dos CPs na Atenção Primária de Saúde, para que os pacientes que receberem alta hospitalar possam receber todo o auxílio e suporte com qualidade em domicílio.

Palavras-chave: cuidados paliativos; terminalidade da vida; unidade de cuidados prolongados; interdisciplinaridade.

\begin{abstract}
Palliative Care $(\mathrm{CP})$ is an approach that promotes relief of suffering for people with incurable diseases that threaten the continuity of life. The objective of this study was to trace the characteristics of patients with CP indication in a long term care unit (UCP). This is a cross-sectional, quantitative, retrospective study with data collection from medical records. During the study period, there were 389 admissions to the UCP, and, of these, 31 medical records were included for this study. The elderly population made up more than $80 \%$ of the sample; $54.8 \%$ were women, mainly affected by chronic diseases related to the aging process, such as neurodegenerative diseases and with short-term hospitalization; and $84 \%$ died. There was a need to strengthen PC in Primary Health Care so that patients who are discharged from the hospital can receive all help and support with quality at home.
\end{abstract}

Keywords: palliative care; terminality of life; long term care unit; interdisciplinarity.

Resumen: Cuidado paliativo (CP) es un enfoque que promueve el alivio del sufrimiento de las personas con enfermedades incurables que amenazan la continuidad de la vida. El objetivo de este estudio fue rastrear las características de los pacientes con indicación de PC en una unidad de atención a largo plazo (UCP). Este es un estudio transversal, cuantitativo, retrospectivo y con recopilación de datos de registros médicos. Durante el período de estudio, hubo 389 ingresos a la UCP y, de estos, se incluyeron 31 registros médicos para este estudio. La población de ancianos constituía más del $80 \%$ de la muestra; el 54,8\% eran mujeres, principalmente afectadas por enfermedades crónicas relacionadas con el proceso de envejecimiento, como las enfermedades neurodegenerativas y con hospitalización a corto plazo; y el 84\% falleció. Es necesario fortalecer los CPs en la Atención Primaria de Salud para que los pacientes dados de alta del hospital puedan recibir toda la ayuda y apoyo con calidad en el hogar.

Palabras clave: cuidados paliativos; terminalidad de la vida; unidad de cuidados a largo plazo; interdisciplinariedad. 


\section{INTRODUÇÃO}

Historicamente, os cuidados paliativos (CPs) surgiram oficialmente em 1960, como uma prática na área da atenção à saúde com o objetivo de cuidar. Consiste em uma abordagem ampla, que visa promover a qualidade de vida de pacientes e familiares que apresentam uma doença crônica incurável, sem possibilidade de tratamentos que possam modificar o curso natural da doença. É baseado em princípios como a prevenção e o alívio de sintomas desagradáveis. Tem como pioneira a médica, enfermeira e assistente social Cicely Saunders, que descreveu o conceito de dor total, envolvendo as dimensões física, social, psicológica e espiritual.

A primeira definição de CP foi publicada pela Organização Mundial da Saúde em 1990 e, em 2002, foi revisada e conceituada como "uma abordagem que promove a qualidade de vida de pacientes e seus familiares, que enfrentam doenças que ameacem a continuidade da vida, por meio da prevenção e do alívio do sofrimento. Requer identificação precoce, avaliação e tratamento da dor e outros problemas de natureza física, psicossocial e espiritual" (WORLD HEALTH ORGANIZATION, 2002).

Mais de 100 milhões de pessoas se beneficiam dos cuidados paliativos anualmente, entretanto, dos que necessitam, menos de $8 \%$ têm acesso garantido nessa forma de assistência e, infelizmente, a formação em cuidados paliativos ainda se encontra distante ou insuficiente no currículo educacional na formação de profissionais de saúde.

A Academia Nacional de Cuidados Paliativos estabeleceu os critérios para elegibilidade nos CPs e o que mais se discute é o tempo de vida do paciente. Foram estabelecidos os critérios como: paciente com doença incurável, em que ele se exime dos tratamentos prolongadores da vida; presença de sintomas desagradáveis não controlados ou dor por mais de 24 horas; internação prolongada sem melhora; prognóstico reservado; entre outros. Existem instrumentos capazes de avaliar o prognóstico de vida através da capacidade funcional, bem como ferramentas para avaliar a indicação de CPs específicos por doenças e condições clínicas.

Para uma abordagem integral do paciente é necessária a atuação de equipe interdisciplinar, composta por no mínimo um médico, um enfermeiro, 
um assistente social ou psicólogo. Pode contar também com fisioterapeuta, farmacêutico e capelão de caráter ecumênico, além da ação intermitente de outros profissionais, que devem ser capacitados e com atendimento exclusivo para a assistência em CP, realizando uma abordagem individualizada e que não se baseia em protocolos.

Ressalta-se que no âmbito dos CPs há quatro modalidades de atuação, sendo: a ação paliativa em que há a abordagem dos sintomas desagradáveis com cuidados prestados desde a atenção básica e sem equipe especializada; CP de grau I - cuidados fornecidos por equipe especializada que não possuem estrutura de atendimento próprio para CP e podem ser oferecidos tanto em nível hospitalar como no domiciliar; CP de grau II - conta com equipe especializada que possui local de internação própria em média complexidade; e CP de grau III - compreende unidades de excelência e de referência em $C P$, envolve capacitação e formação de profissionais na área.

Nos últimos anos, a prática de cuidados paliativos vem se expandindo, visando também otimizar a funcionalidade do indivíduo independentemente do prognóstico, incentivando a qualidade de vida com a reabilitação em novas modalidades, com exercícios, grupos de dança e yoga como aliados na redução de sintomas, a fim de proporcionar o bem-estar geral para os pacientes e mudar o paradigma de que todo paciente em cuidados paliativos é acamado e debilitado.

No Brasil, o Ministério da Saúde normatizou os cuidados paliativos através da Resolução n. 41, de 31 de outubro de 2018, que dispõe sobre as diretrizes para a organização dos cuidados paliativos, fazendo parte dos Cuidados Continuados Integrados (CCl), no âmbito do Sistema único de Saúde (SUS), e afirma que esses devem ser ofertados na atenção básica, domiciliar e ambulatorial, urgência e emergência e na atenção hospitalar, sendo um avanço necessário para os CPs no nosso país.

Outro local de assistência são as Unidades de Cuidados Prolongados (UCP), que prestam cuidados em nível intermediário entre os hospitais de pacientes agudos e a atenção primária, posterior ao retorno do paciente ao domicílio, destinando-se a usuários com situação clínica estável que necessitem de reabilitação clínica funcional e prestando assistência como 
retaguarda para os que necessitam de cuidados paliativos. Este estudo foi realizado em uma UCP.

Diante deste cenário, este estudo justifica-se em função da necessidade de conhecimento do público em cuidados paliativos, com vista a ampliar as discussões a respeito do tema, haja vista que já acontecem no local deste estudo, além de fomentar a estruturação de uma equipe multiprofissional especializada e preparada para atuação em cuidados paliativos. O objetivo deste estudo foi traçar as características dos pacientes com indicação de cuidados paliativos em uma unidade de cuidados prolongados (UCP) no Hospital São Julião de Campo Grande, Mato Grosso do Sul (MS).

\section{MÉTODOS}

Este é um estudo retrospectivo, transversal e com abordagem quantitativa, realizado em uma UCP que conta com 32 leitos de retaguarda no Hospital São Julião, no município de Campo Grande, MS. Foram incluídos no estudo pacientes com indicação para cuidados paliativos com identificação nos prontuários, através dos termos "cuidados paliativos" ou "cuidados paliativos exclusivos" ou "cuidados paliativos não exclusivos", com idade mínima de 18 anos, internados no período de abril de 2017 a abril de 2018. Os critérios de exclusão foram prontuários de pacientes sem indicação para cuidados paliativos, de acordo com as informações fornecidas nos prontuários no período predeterminado, pacientes com idade inferior a 18 anos e prontuários com informações insuficientes.

A coleta dos dados foi realizada a partir da revisão dos prontuários e da análise das informações e evoluções diárias. Foram coletados dados referentes à idade, sexo, escolaridade, estado civil, religião, doença, tempo de internação e desfecho clínico. Após a coleta, os dados foram tabulados em planilhas do programa Microsoft Excel ${ }^{\circledR} 2010$ e os resultados expressos pela análise descritiva simples.

Foi solicitada a dispensa do Termo de Consentimento Livre e Esclarecido (TCLE), por se tratar de uma pesquisa com contato somente com os prontuários como base de dados, sem acesso direto ao paciente. O estudo foi submetido e aprovado pelo Comitê de Ética em Pesquisa com 
Seres Humanos da Universidade Federal de Mato Grosso do Sul (CEP/UFMS), sob o Parecer n. 2.685.974 de 30/05/2018.

\section{RESULTADOS}

No período do estudo, foram admitidos na UCP 389 pacientes; destes, após a análise dos prontuários e considerados os critérios de inclusão para o estudo, 31 prontuários foram elegidos para a pesquisa, perfazendo $8 \%$ da população admitida.

A Tabela 1 descreve as características sociodemográficas dos participantes, sendo $54,8 \%(n=17)$ do sexo feminino e $45,2 \%(n=14)$ do sexo masculino, com média de idade de 79,3 anos $( \pm 19,7)$, variando entre idade mínima e máxima de 27 e 100 anos, respectivamente; 65,5\% ( $n=20)$ tinham idade $\geq 80$ anos.

Os pacientes receberam a indicação para cuidados paliativos variando desde o primeiro dia de internação até o 65 dia; 19,4\% dos participantes foram admitidos na UCP já em cuidados paliativos; 48,4\% receberam a indicação na primeira semana de internação; 16,1\% foram indicados para cuidados paliativos na terceira semana de internação; e 16,1\% na terceira semana em diante.

Tabela 1 - Número e porcentagem de participantes segundo sexo, faixa etária, estado civil, escolaridade e religião

\begin{tabular}{lcc}
\hline \multicolumn{1}{c}{ Variáveis } & N. & $\%$ \\
\hline Sexo & & \\
Feminino & 17 & 54,8 \\
Masculino & 14 & 45,2 \\
Faixa etária & & \\
27 a 59 anos & 5 & 16,1 \\
60 a 79 anos & 6 & 19,4 \\
$\geq 8$ anos & 20 & 64,5 \\
Estado civil & & \\
Solteiro & 8 & 25,8 \\
Casado & 6 & 19,4 \\
Divorciado & 1 & 3,2
\end{tabular}




\begin{tabular}{lcc}
\hline \multicolumn{1}{c}{ Variáveis } & N. & \% \\
\hline Viúvo & 14 & 45,2 \\
Amasiado & 1 & 3,2 \\
Sem informação* & 1 & 3,2 \\
Escolaridade & & \\
Analfabeto & 16 & 51,6 \\
Fundamental completo & 4 & 13 \\
Fundamental incompleto & 8 & 25,8 \\
Ensino Médio completo & 1 & 3,2 \\
Ensino Superior completo & 1 & 3,2 \\
Sem informação* & 1 & 3,2 \\
Religião & & \\
Católico & 16 & 51,6 \\
Evangélico & 6 & 19,4 \\
Nenhuma & 8 & 25,8 \\
Sem informação* & 1 & 3,2 \\
\hline
\end{tabular}

Nota: *sem descrição dos dados no prontuário.

Fonte: Elaboração própria.

A doença mais frequente registrada nos prontuários dos pacientes com indicação de cuidados paliativos foi a hipertensão arterial sistêmica, com alta prevalência em ambos os sexos, compondo 44,4\% ( $n=8)$ e $26,7 \%$ ( $n=4)$ nas mulheres e homens, respectivamente. As doenças neurodegenerativas também tiveram destaque, como a doença de Parkinson e Alzheimer, compostas por $35,4 \%(n=11)$; seguidas pelas afecções pulmonares, com $25,8 \%$ $(n=8)$; doenças cerebrovasculares exclusivamente causadas por acidente vascular cerebral, variando sua forma entre isquêmico e hemorrágico, com $12,9 \%(n=4)$, sendo mais frequente no sexo feminino; e doenças oncológicas, com 12,9\% $(n=4)$ (Tabela 2$)$. 
Tabela 2- Número e porcentagem de doenças segundo sexo

\begin{tabular}{|c|c|c|c|c|c|c|}
\hline \multirow{3}{*}{ Doenças } & \multicolumn{4}{|c|}{ Sexo } & & \\
\hline & \multicolumn{2}{|c|}{$\begin{array}{c}\text { Feminino } \\
(\mathrm{n}=17)\end{array}$} & \multicolumn{2}{|c|}{$\begin{array}{l}\text { Masculino } \\
(n=14)\end{array}$} & \multicolumn{2}{|c|}{ Total } \\
\hline & № & $\%$ & № & $\%$ & № & $\%$ \\
\hline Diabetes Mellitus & 1 & 5,6 & 3 & 20 & 4 & 12,1 \\
\hline Doença coronariana & 0 & 0 & 1 & 6,7 & 1 & 3 \\
\hline Hipertensão arterial sistêmica & 8 & 44,4 & 4 & 26,7 & 12 & 36,4 \\
\hline Afecções pulmonares & 4 & 23,5 & 4 & 28,6 & 8 & 25,8 \\
\hline Cerebrovasculares & 3 & 17,6 & 1 & 7,1 & 4 & 12,9 \\
\hline Infectocontagiosas & 1 & 5,9 & 1 & 7,1 & 2 & 6,5 \\
\hline Neurodegenerativas & 6 & 35,3 & 5 & 35,8 & 11 & 35,4 \\
\hline Neurológicas & 1 & 5,9 & 1 & 7,1 & 2 & 6,5 \\
\hline Oncológicas & 2 & 11,8 & 2 & 14,3 & 4 & 12,9 \\
\hline
\end{tabular}

Nota: *Admite mais de uma doença por participante.

Fonte: Elaboração própria.

Em relação ao tempo de internação, este variou de 1 a 122 dias, apresentando tempo médio de 22,8 dias $( \pm 26,4)$ (Figura 1$)$, tendo como desfecho clínico um alto índice de óbitos (Figura 2).

Figura 1 - Tempo de internação em dias na Unidade de Cuidados Prolongados segundo sexo

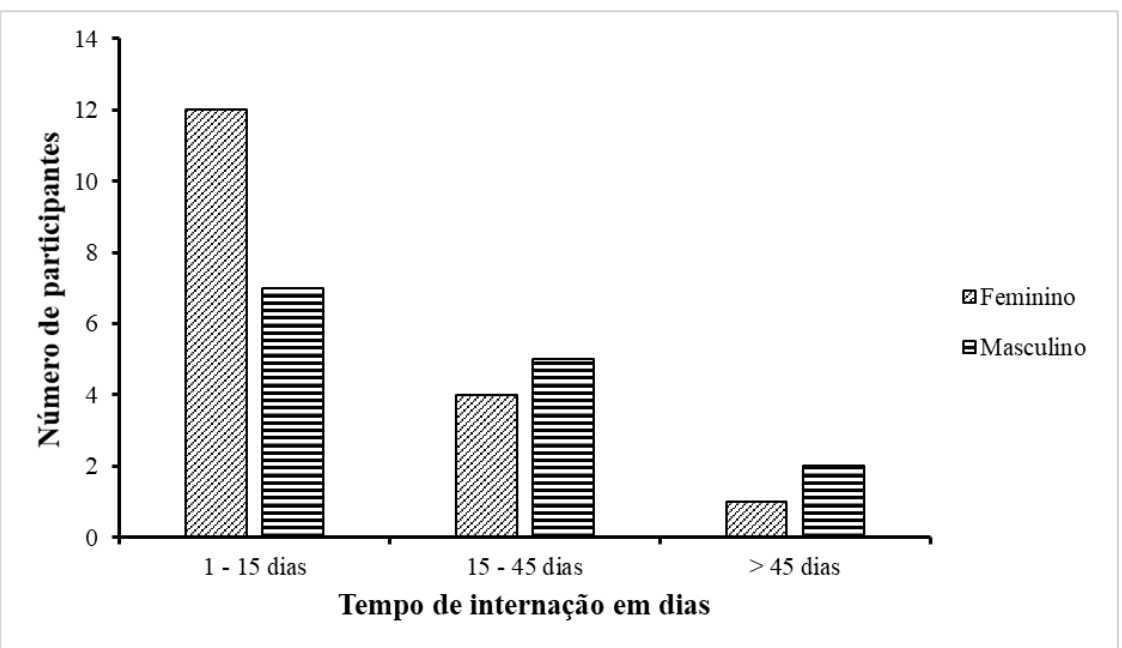

Fonte: Elaboração própria. 
Figura 2 - Desfecho clínico dos pacientes com indicação de cuidados paliativos

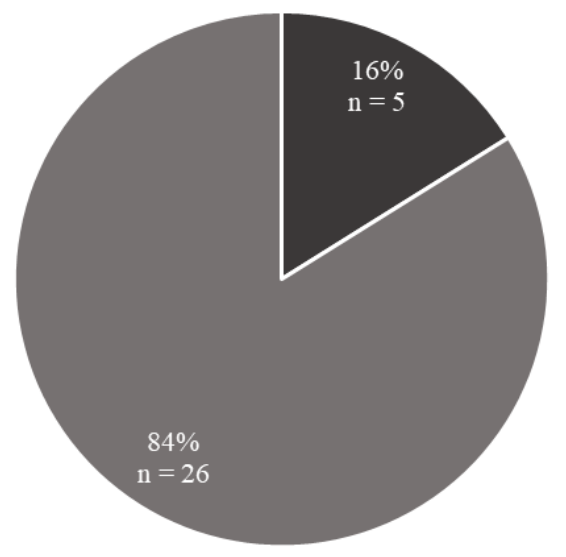

- Alta - Óbito

Fonte: Elaboração própria.

\section{DISCUSSÃO}

Ao longo do século XX, iniciou-se um importante processo de transição demográfica no mundo, com a queda na taxa de mortalidade, a diminuição das doenças de origem infecciosa e a redução da natalidade. Além disso, os avanços tecnológicos e de acesso na área da saúde contribuíram para um aumento da expectativa de vida da população (KALACHE, 2008; CARMO; DAGNINO; JOHANSES, 2014).

Contudo, com a mudança de perfil das doenças nesse processo de transição, com a queda das doenças infecciosas com alta letalidade e aumento das doenças crônicas degenerativas, tornou-se possível que as pessoas vivam mais e atinjam a longevidade, porém isso acarreta grandes desafios e novas demandas para os sistemas de saúde. No entanto, apesar de todos os avanços na saúde, a morte permanece como um ciclo natural da vida, que não deve ser prolongado nem antecipado (ONU, 2012; MATSUMOTO, 2012; KALACHE, 2008).

O processo de adoecimento pode repercutir de diversas formas na vida dos pacientes e seus familiares, ainda mais quando há um prognóstico 
reservado. Nessa perspectiva, os cuidados paliativos atuam com o alívio de sofrimento, não apenas o físico, mas em todas as dimensões. Essa abordagem deve ser iniciada de forma precoce, respeitando a autonomia nas escolhas e decisões do paciente e permitindo que ele tenha uma morte digna (MACHADO et al., 2019; ANTUENO; SILBERBERG, 2018; KOVÁCS, 2014).

Os resultados deste estudo demonstraram que o público que mais recebeu indicações para cuidados paliativos na Unidade de Cuidados Prolongados foi o de idosos, compondo 83,9\% da amostra; destaca-se a média de idade de 79,3 anos, sendo que mais da metade dos idosos do estudo apresentavam idade igual ou maior que 80 anos. Bastos et al. (2018) mostrou que a faixa etária mais frequente no seu estudo foi acima de 60 anos, compondo $38,9 \%$ da amostra. Esse fato se relaciona com a diminuição progressiva na capacidade de regeneração e divisão celular, pela imunosenescência com o declínio progressivo da imunidade associado à idade e também pelo aumento da expectativa de vida resultante do processo de transição demográfica que está relacionada ao aumento das doenças crônicas degenerativas (ANTUNES et al., 2015; MARTORANA et al., 2012).

Costa et al. (2016) concluem que, com a longevidade em progressão, há a necessidade de melhorar o acesso para os idosos, oferecendo dignidade e qualidade de vida, sendo este grupo populacional um dos mais fragilizados da sociedade. Para Gomes et al. (2011), a população que mais tem necessitado dos CPs são os idosos. Gardiner et al. (2011) observaram o deficit no encaminhamento de idosos com doença crônica incurável para serviços especializados em CP, sendo que os idosos com doença oncológica possuem maior probabilidade de serem encaminhados para esses serviços devido à ligação histórica dos CPs com o câncer.

Pessoas do sexo feminino $(54,8 \%)$ receberam mais indicação para cuidados paliativos na Unidade de Cuidados Prolongados, assim como em estudo realizado em uma enfermaria de CP no Recife, realizado por Oliveira et al. (2018), em que foi observado que mais da metade dos pacientes internados eram do sexo feminino (53\%). Souza, Simão e Lima (2012) justificam esses resultados devido à prevalência do sexo feminino com diagnóstico de câncer e indicam que, apesar de elas viverem mais, adoecem mais do que em comparação aos homens. 
Quanto à escolaridade, observa-se que mais de $50 \%$ da amostra foi composta por analfabetos. Em um estudo de Souza, Simão e Lima (2012) envolvendo 239 pacientes em cuidados paliativos, o nível de escolaridade mais prevalente foi o Ensino Fundamental, com 58,5\%, seguido do Ensino Médio. Bastos et al. (2018) identificaram que a maioria apresentava apenas o Ensino Fundamental incompleto. Schneider e D’Orsi (2009) observaram que mulheres analfabetas apresentam maior risco de óbito do que as com nível superior. Diante dos estudos supracitados, podemos deduzir que a baixa escolaridade pode estar aliada a piores condições de saúde, com diagnóstico de doenças em fases mais avançadas, podendo, assim, comprometer o seu desfecho clínico.

Cerca de $71 \%$ dos participantes desse estudo apresentavam alguma filiação religiosa, resultado esse semelhante ao de Bastos et al. (2018), com a religião católica como a mais comum entre os participantes; 25,8\% ( $n=8)$ da amostra deste estudo não apresentou religião, não descartando a possibilidade de serem espiritualizados, o que consiste na crença/busca de um ser transcendente, na busca pelo sentido da vida e que nem sempre está interligada com a religiosidade (ARRIEIRA et al., 2018).

Arrieira et al. (2017) conclui que a espiritualidade proporciona aproximação e vínculo entre os pacientes em cuidados paliativos e os profissionais que fazem parte da equipe de assistência. Para que se alcance a integralidade do cuidado em sua totalidade, é necessária a inclusão do cuidado espiritual em todo esse processo de terminalidade e no suporte aos familiares em todas as fases até o luto.

A religião pode reduzir o sofrimento envolvido no processo de finitude, auxiliando na compreensão das incertezas da vida, e influencia muito em situações estressantes, como no enfrentamento de doenças que ameacem a continuidade da vida, sendo utilizada como um recurso para encarar e lidar com a doença. Neste contexto, a religiosidade pode proporcionar respostas para os questionamentos envolvidos nos acontecimentos da vida, como o adoecimento, possibilitando a reconstrução do contexto de conviver com uma doença (SAPORETTI; SILVA, 2012; BARBOSA; FREITAS, 2009).

De todos os pacientes, 51,5\% apresentavam comorbidades como hipertensão arterial, diabetes mellitus, doença coronariana associada à doença 
principal para critérios de indicação para CP. Faria et al. (2015) apontaram que $28,1 \%$ dos pacientes em cuidados paliativos apresentaram doenças relacionadas a dois ou mais sistemas diferentes e a principal causa de indicação dos cuidados paliativos foram as doenças cardiovasculares. Segundo Arantes (2012), os idosos, principalmente, podem apresentar doenças crônicas simultâneas, como a hipertensão arterial e diabetes, embora essas não possuam nenhuma condição fatal iminente e também, isoladamente, não sejam condições convencionais para a indicação de cuidados paliativos.

Apenas 12,9\% apresentaram doença oncológica, sendo destaque as doenças neurodegenerativas, que são frequentemente atreladas ao processo de envelhecimento. Marcucci et al. (2016) e Arcanjo et al. (2018) demostraram que as principais doenças encontradas foram as não oncológicas, sendo o câncer uma patologia muito relacionada aos critérios de elegibilidade para os cuidados paliativos, porém tendo como predomínio as doenças cerebrovasculares, demência avançada, doença pulmonar obstrutiva crônica (DPOC), doença renal, insuficiência cardíaca congestiva (ICC), entre outras, o que está relacionado com os resultados deste estudo.

Durante a internação, observamos que o tempo médio de permanência no hospital foi de 22,8 dias, em que $84 \%$ do desfecho clínico foi composta pelo óbito. Estes dados concordam com Oliveira et al. (2018), que apresentaram característica de internação em curto prazo com média de 18 dias e com 55\% dos pacientes evoluindo para o óbito, sendo a morte um evento natural e esperado na presença de doença incurável, em que não há possibilidades terapêuticas para a cura. Arcanjo et al. (2018) concluíram que o alto índice de mortalidade de pacientes em cuidados paliativos no ambiente hospitalar sugere que tais cuidados foram implementados próximos ao fim do curso da doença; acreditam ainda que, quanto mais precoce a implementação dos CPs e o seguimento dos mesmos cuidados na Atenção Primária de Saúde (APS), maiores os benefícios aos pacientes com esse modelo de assistência.

A respeito dos pacientes em CP que recebem alta hospitalar e necessitam da continuidade dessa linha de cuidados em domicílio, neste estudo, foi apontado que $16 \%$ da amostra teve como desfecho clínico a alta da internação hospitalar e possivelmente não houve o acompanhamento 
de uma equipe especializada. A integralidade dos serviços por meio dos processos de referência e contrarreferência é essencial para assegurar uma assistência de qualidade e para que esse paciente/família seja visto e acompanhado de maneira adequada e humanizada (MOLINA et al., 2006). Estudos mostram que o serviço ofertado pelas equipes de APS em domicílio é a preferência dos pacientes e familiares, e a morte em domicílio é considerada um indicador de qualidade em cuidados paliativos (BARNES et al., 2007; NEERGAARD et al., 2010).

\section{CONSIDERAÇÕES FINAIS}

Este estudo permitiu conhecer mais detalhadamente as características dos pacientes que recebem indicação de cuidados paliativos na Unidade de Cuidados Prolongados do Hospital São Julião. Destacaram-se os idosos, compondo mais de $80 \%$ de toda a amostra, acometidos por doenças crônicas relacionadas principalmente ao processo de envelhecimento, como as doenças neurodegenerativas. Observamos o alto índice de óbito, que é um evento natural e esperado em um indivíduo com uma doença incurável, porém, de acordo com o que foi discutido na literatura, isso pode sugerir que os cuidados paliativos estejam sendo iniciados próximos ao fim de vida, haja vista que a abordagem dos CPs deve ser iniciada de forma precoce, independentemente do prognóstico do tempo de vida restante. Adverte-se a necessidade da estruturação de uma equipe interdisciplinar preparada para atuar com uma indicação mais precisa para CP com auxílio de instrumentos validados na literatura, a fim de lidar com os pacientes e familiares oferecendo melhor assistência, qualidade de vida e dignidade nesse processo de terminalidade. É necessário o fortalecimento dos cuidados paliativos na Atenção Primária de Saúde, para que os pacientes que obtiverem alta hospitalar possam receber todo o auxílio e suporte com qualidade. Este estudo poderá contribuir para pesquisas futuras na Unidade de Cuidados Prolongados. 


\section{REFERÊNCIAS}

ANTUENO, P.; SILBERBERG, A. Eficacia de los cuidados paliativos en el alivio del sufrimiento. Persona y bioética, Chía, v. 22, n. 2, p. 367-80, 2018.

ANTUNES, Y. P. P. V.; BUGANO, D. D. G.; GIGLIO, A. D; KALIKS, R. A.; KARNAKIS, T.; PONTES, L. B. Características clínicas e de sobrevida global em pacientes oncológicos idosos num centro oncológico terciário. Einstein, São Paulo, v. 13, n. 4, p. 487-91, 2015.

ARANTES, A. C. L. Q. Indicação de cuidados paliativos. In: CARVALHO, R. T.; PARSONS, H. A. (Org.) Manual de cuidados paliativos. São Paulo: Academia Nacional de Cuidados Paliativos (ANCP), 2012. p. 56-74.

ARCANJO, S. P.; SAPORETTI, L. A.; CURIATI, J. A. E; JACOB-FILHO, W.; AVELINO-SILVA, T. J. Características clínicas e laboratoriais associadas à indicação de cuidados paliativos em idosos hospitalizados. Einstein, São Paulo, v. 16, n. 1, p. 1-8, 2018.

ARRIEIRA, I. C. O.; THOFERHN, M. B.; SCHAEFER, O. M.; FONSECA, A. D.; KANTORSKI, L. P.; CARDOSO, D. H. O sentido do cuidado espiritual na integralidade da atenção em cuidados paliativos. Revista Gaúcha de Enfermagem, Porto Alegre, v. 38, n. 3, p. 1-9, 2017.

ARRIEIRA, I. C. O.; THOFERHN, M. B.; PORTO, A. R.; MOURA, P. M. M.; MARTINS, C. L.; JACONDINO, M. B. Spirituality in palliative care: experiences of an interdisciplinary team. Revista da Escola de Enfermagem da USP, São Paulo, v. 52, p. 1-7, 2018.

BARBOSA, K. A.; FREITAS, M. H. Religiosidade e atitude diante da morte em idosos sob cuidados paliativos. Revista Kairós, São Paulo, v. 12, n. 1, p. 113-34, 2009.

BARNES, E. A.; FAN, G.; HARRIS, K.; DOYLE, M.; LIBRACH, L. S.; CHOW, E.; BARBERA, L.; TSAO, M.; LAM, K.; DANJOUX, C. Involvement of family physicians in the care of cancer patients seen in the Palliative Rapid Response Radiotherapy Program. Journal of Clinical Oncology, v. 25, n. 36, p. 5758-62, 2007.

BAStOS, B. R.; PEREIRA, A. K. S.; CASTRO, C. C.; CARVAlho, M. M. C. Perfil sociodemográfico dos pacientes em cuidados paliativos em um hospital de referência em oncologia do estado do Pará, Brasil. Revista Pan-Amazônica de Saúde. v. 9, n. 2, p. 31-36. 2018.

CARMO, R. S.; DAGNINO, R. S.; JOHANSEN, I. C. Demographic transition and transition of urban water consumption in Brazil. Revista Brasileira de Estudos de População, v. 31, n. 1, p. 170-7, 2014. 
COSTA, R. S.; SANTOS, A. G. B.; YARID, S. D; SENA, E. L. S.; BOERY, R. N. S. O. Reflexões bioéticas acerca da promoção de cuidados paliativos a idosos. Saúde em debate. v. 40, n. 108, p. 170-7. 2016.

FARIA, J. A. M.; FERREIRA, L. G.; VIEIRA, M. A. B; COSENZA, N. N.; ALVARENGA, P. P.; FIGUEIREDO, P. L. Perfil de pacientes com indicação de cuidados paliativos internados no Hospital Júlia Kubitschek - FHEMIG. Revista Médica de Minas Gerais, Belo Horizonte, v. 25, n. 1, p. 25-9, 2015.

GARDINER, C.; COBB, M.; GOTT, M.; INGLETON, C. Barriers to providing palliative care for older people in acute hospitals. Age and Ageing, v. 40, n. 2, p. 233-8, 2011.

GOMES, B.; COHEN, J.; DELIENS, M; HIGGINSON, I. International trends in circumstances of death and dying among older people. In: GOTT, M.; INGLETON, C. (Ed.). Living with ageing and dying: palliative and end of life care for older people. 1. ed. Inglaterra: Oxford University Press, 2011, p. 3-18.

KALACHE, A. O mundo envelhece: é imperativo criar um pacto de solidariedade social. Ciência e Saúde Coletiva, Rio de Janeiro, v. 13, n. 4, p. 1107-11, 2008.

KOVÁCS, M. J. A caminho da morte com dignidade no século XXI, Revista Bioética, Brasília, DF, v. 22, n. 1, p. 94-104, 2014.

MACHADO, J. C.; REIS, H. F. T.; SENA, E. L. S.; SILVA, R. S. S.; BOERY, R. N. S. O.; VILELA, A. B. A. El fenómeno de la conspiración del silencio en pacientes en cuidados paliativos: una revisión integrativa. Revista Enfermería Actual, San José, v. 36, p. 1-12, 2019.

MARCUCCI, F. C. I.; PERILLA, A. B.; BRUN, M. M.; CABRERA, M. A. S. Identificação de pacientes com indicação de Cuidados Paliativos na Estratégia Saúde da Família: estudo exploratório. Cadernos Saúde Coletiva, Rio de Janeiro, v. 24, n. 2, p. 14552, 2016.

MARTORANA, A.; BULATI, M.; BUFFA, S.; PELLICANÒ, M.; CARUSO, C.; CANDORE, G.; COLONNA-ROMANO, G. Immunosenescence, inflammation and Alzheimer's disease. Longevity \& Healthspan, v. 1, n. 1, p. 1-8, 2012.

MATSUMOTO, D. Y. Cuidados Paliativos: conceito, fundamentos e princípios. In: CARVALHO, R. T.; PARSONS, H. A. (Org.). Manual de cuidados paliativos. São Paulo: Academia Nacional de Cuidados Paliativos (ANCP), 2012, p. 23-30. 
MOLINA, E. H.; GIL, J. R.; PINNA, M. A. C.; MORALO, M. J. R. Primer nivel asistencial en cuidados paliativos: evolución del contenido de la cartera de servicios de atención primaria y criterios de derivación al nivel de soporte. Atención Primaria, v. 38, n. 2, p. 85-92. 2006.

NEERGAARD, M. A.; VEDSTESD, P.; OLESEN, F.; SOKOLOWSKI, I.; JENSEN, A.; SONDERGAARD, J. Associations between successful palliative trajectories, place of death and GP involvement. Scandinavian Journal of Primary Health Care, v. 28, n. 3, p. 138-45, 2010.

OLIVEIRA, A. G.; RIBEIRO, S. Z. R. S.; SILVA, M. I. C.; VIDAL, S. A.; LOPES, L. G. F. Perfil das internações em cuidados paliativos: uma ferramenta à gestão. Revista de Enfermagem UFPE, Recife, v. 12, n. 8, p. 2082-8, 2018.

ONU. Envelhecimento no século XXI: celebração e desafio- resumo executivo. Nova York: Fundo de População das Nações Unidas (UNFPA): Help Age International, 2012.

SAPORETTI, L. A.; SILVA, A. M. O. P. Aspectos particulares e ritos de passagem nas diferentes religiões. In: CARVALHO, R. T.; PARSONS, H. A. Manual de cuidados paliativos - ANCP. 2. ed. ampl. atual. [S.I.]: ANCP, 2012.

SCHNEIDER, I. J. C.; D'ORSI, E. Sobrevida em cinco anos e fatores prognósticos em mulheres com câncer de mama em Santa Catarina, Brasil. Caderno de Saúde Pública, Rio de Janeiro, v. 25, n. 6, p. 1285-96, 2009.

SOUZA, R. S.; SIMÃO, D. A. S.; LIMA, E. D. R. Perfil sociodemográfico e clínico de pacientes atendidos em um serviço ambulatorial de quimioterapia paliativa em Belo Horizonte. Revista Mineira de Enfermagem, Belo Horizonte, v. 16, n. 1, p. 38-47, 2012.

WORLD HEALTH ORGANIZATION. National cancer control programmes: policies and managerial guidelines. 2. ed. Geneva: World Health Organization, 2002. 